\title{
Prevalence of diabetic retinopathy in patients with diabetes mellitus diagnosed after the age of 70
}

\author{
years
}

M Cahill, A Halley, M Codd, N O’Meara, R Firth, D Mooney, R W Acheson

Institute of

Ophthalmology, Mater

Misericordiae

Hospital, Eccles

Street, Dublin 7

M Cahill

A Halley

$\mathrm{R}$ W Acheson

Department of Epidemiology and

Health Services

Research, Mater

Misericordiae

Hospital, 58 Eccles

Street, Dublin 7

M Codd

Department of Endocrinology, Mater

Misericordiae

Hospital, Dublin 7

N O’Meara

R Firth

The Royal Victoria Eye and Ear Hospital,

Adelaide Road,

Dublin 2

M Cahill

D Mooney

Correspondence to: Mr Robert Acheson, Institute of Ophthalmology, Mater Misericordiae Hospital, Eccles Street, Dublin 7, Ireland.

Accepted for publication 6 January 1997

\begin{abstract}
Aims/background-A hospital based prevalence study was undertaken to estimate the prevalence of diabetic retinopathy (DR) in patients diagnosed as having diabetes mellitus after the age of 70 years. The prevalence of visually threatening retinopathy at the time of diagnosis of diabetes was also determined. The association between prevalence of $D R$ and duration of diabetes mellitus, mode of treatment, $\mathrm{HbA}_{1 \mathrm{c}}$ levels, presence of hypertension, and sex of patient was examined and a comparison was drawn between this study and earlier prevalence studies of DR in older type II diabetics. Methods-Using data on the Irish Diabetic Retinopathy Register located in the Mater Misericordiae Hospital, Dublin, all patients who were diagnosed as having type II diabetes mellitus after the age of 70 years were invited to attend for ophthalmic review. Medical records were examined to determine the duration of diabetes mellitus, mode of treatment, recent $\mathrm{HbA}_{1 \mathrm{c}}$ levels, and the presence of systemic hypertension.
\end{abstract}

Results-Of the 150 patients examined, 21 (14\%) had some form of DR and 10 of these patients $(6.6 \%)$ had visually threatening retinopathy or previously treated visually threatening retinopathy. Five patients $(3.3 \%)$ presented with visually threatening retinopathy at the time of diagnosis of diabetes. Those patients with DR had a significantly higher median duration of diabetes (5.0 years) compared with those patients without DR (3.5 years). A significantly higher proportion of patients with DR required treatment with insulin and a correspondingly lower proportion of patients without DR were controlled on diet alone. There was no significant association between prevalence of $D R$ and $H b A_{1 c}$ levels, systemic hypertension, or sex of patient. There was a lower overall prevalence of $D R$ in comparison with earlier studies.

Conclusions-The prevalence of DR in these elderly type II diabetics is lower than that previously reported in patients with type II disease but a small percentage of patients had visually threatening retinopathy at presentation. Longer duration of diabetes and insulin use were associated with a significantly increased prevalence of DR. All elderly type II diabetic patients require thorough ophthalmic examination near to the time of first presentation and thereafter at regular intervals.

(Br F Ophthalmol 1997;81:218-222)

Diabetic eye complications and, in particular, diabetic retinopathy are a leading cause of blindness in industrialised countries. ${ }^{1}$ Type I diabetes mellitus is more commonly seen in younger patients and requires treatment with insulin. Type II diabetes is seen in older patients and can in most cases be treated with diet modification alone, or in combination with oral hypoglycaemic agents (although in some cases insulin therapy is required). The prevalence of diabetic retinopathy is higher in type I diabetics than in those with type II disease. ${ }^{2}$

Previous prevalence studies of diabetic retinopathy (DR) have grouped patients according to factors such as age at diagnosis, ${ }^{2-5}$ type of diabetes mellitus, ${ }^{67}$ disease duration, ${ }^{8}$ ethnic background, ${ }^{9-13}$ or a combination of these factors. ${ }^{14-16}$ However, patients with type II diabetes do not form a homogeneous group and may have ages (at the time of diagnosis) ranging from 30 to 70 years or more. While no previous study has concentrated on the prevalence of DR in patients diagnosed after the age of 70 years, three earlier prevalence studies have noted that older type II diabetic patients have a lower prevalence of DR in comparison with their younger counterparts. ${ }^{35} 16$

This study was designed to determine the prevalence of DR in patients with diabetes mellitus diagnosed after the age of 70 years in a hospital based Irish population and to estimate the percentage of patients who had visually threatening DR at the time of diagnosis of diabetes mellitus. The association between prevalence of DR and duration of diabetes, mode of treatment, haemoglobin $\mathrm{A}_{1 \mathrm{c}}\left(\mathrm{HbA}_{1 \mathrm{c}}\right)$ levels, systemic hypertension, and sex of patient was examined and a comparison made between the findings of this study and those of previous prevalence studies which included older type II diabetics.

Patients and methods

The Mater Misericordiae Hospital has had established services for diabetic patients for the past 30 years including a diabetic day centre and a separate, dedicated diabetic chart system which is used in close conjunction with the normal medical chart system. The diabetic service has close links with the ophthalmology 
department including a retinopathy screening clinic which takes place at the same time as one of the diabetic clinics on a weekly basis. In the Irish Republic, neither family practitioners nor opticians screen for DR. The eye unit is the regional eye centre for the north eastern part of the country, its consultant staff includes a vitreoretinal surgeon and it serves a population of approximately $900000 .^{17}$

The Irish Diabetic Retinopathy Register, established in 1991, is a computerised database located in the Mater Misericordiae Hospital. The database contains demographic, medical, and ophthalmic data recorded prospectively on 4000 patients attending four different hospitals in the eastern region of the Irish Republic. The catchment population for these hospitals is approximately 1.5 million. One hospital is a university linked teaching hospital, another is specialised for eyes and ears, and the other two are district general hospitals. The data are retrieved from the database using a query generator based on diagnostic entities.

Those patients with diabetes mellitus diagnosed after the age of 70 years were identified and contacted by letter inviting them to attend for clinical examination by one of two ophthalmologists (MC and RWA). A second letter was sent to non-responders repeating the first invitation. The clinical examinations were carried out using direct ophthalmoscopy and slit-lamp fundus biomicroscopy. Indirect ophthalmoscopy was available if required. Fluorescein angiography was performed for visually threatening retinopathy. The various stages of DR were classified according to the protocol for screening for DR produced by the Retinopathy Working Party. ${ }^{1}$ Patients with no clinical signs of DR following treatment for maculopathy, preproliferative and proliferative retinopathy were classified as having DR. Maculopathy, preproliferative and proliferative retinopathy were classified as being visually threatening retinopathy. The computer database was used to identify which patients had visually threatening DR when their diabetes was diagnosed, enabling a prevalence figure for this to be determined.

The medical records of participating patients were reviewed to determine the duration of disease, mode of treatment of diabetes, recent $\mathrm{HbA}_{1 \mathrm{c}}$ levels, and the presence of systemic hypertension. The duration of diabetes was calculated as that period from the date the diagnosis was made in a diabetic clinic to the date of the study examination, rounded off to the nearest month. The patients were classified according to their mode of treatment depending on whether they were insulin requiring, controlled on oral hypoglycaemic agents, or controlled on diet alone. $\mathrm{HbA}_{1 \mathrm{c}}$ levels recorded in diabetic charts were utilised if these had been taken within the 3 months before the study examination. The $\mathrm{HbA}_{1 \mathrm{c}}$ levels were estimated using a high performance liquid chromatography method and a value greater than $7.0 \%$ was taken to be abnormally elevated. Patients were deemed to have hypertension if their physician had previously made
Table 1 Prevalence of diabetic retinopathy in patients with diabetes mellitus diagnosed after the age of 70. Classification of type of diabetic retinopathy

\begin{tabular}{lc}
\hline 1 Background retinopathy & 11 \\
2 Clinically significant maculopathy & 2 \\
3 Preproliferative retinopathy & 0 \\
4 Proliferative retinopathy & 1 \\
5 Photocoagulated clinically significant & 3 \\
maculopathy & 0 \\
6 Photocoagulated preproliferative retinopathy & 2 \\
7 Photocoagulated proliferative retinopathy & 2 \\
8 Advanced retinopathy & $21(14.0 \%)$ \\
Total & \\
\hline
\end{tabular}

the diagnosis and had commenced appropriate treatment.

The significance of associations between DR and the previously outlined factors was determined using the Student's $t$ test for normally distributed continuous variables, the MannWhitney $U$ test for continuous variables with a skewed distribution, and the $\chi^{2}$ test for categorical variables. Statistical significance implies $\mathrm{p}<0.05$ unless otherwise stated.

\section{Results}

There were 230 patients registered on the database who had been diagnosed after the age of 70 . Of these, 150 attended for clinical review while 26 were reported to be deceased (response rate $76.5 \%$ ). Of those examined, 94 were female and 56 male (1.7:1). The mean age at examination was 79.8 years (range 70 to 92 years).

PREVALENCE OF DIABETIC RETINOPATHY

Table 1 outlines the relative frequency of the different forms of DR and its overall prevalence in this study group (21 of $150 ; 14 \%$ ). While background retinopathy occurred most frequently $(11 / 21 ; 52 \%), 10$ patients $(6.6 \%)$ were found to have visually threatening retinopathy or previously treated visually threatening retinopathy. Five patients $(3.3 \%)$ had visually threatening retinopathy at presentation. Of these five patients, three had clinically significant maculopathy, while the remaining two were noted to have proliferative retinopathy.

ASSOCIATION BETWEEN DIABETIC RETINOPATHY AND DURATION OF DIABETES

The median duration of diabetes in those with DR (5.0 years; 2.0-10.0 years) was significantly higher than the median duration in those without ( 3.5 years; $0.5-16.4$ years) $(p=$ 0.007).

\section{ASSOCIATION BETWEEN DIABETIC RETINOPATHY} AND MODE OF TREATMENT

There was a significant difference between those with and without DR with respect to mode of treatment required. While a similar proportion of both groups was controlled on oral hypoglycaemic agents ( $57 \%$ v 52\%), a significantly higher proportion of those with DR required insulin $(14 \%, v 2 \%)$ and a correspondingly lower proportion of patients without DR could be managed on diet alone (33\% $v 45 \%$ ) (Table 2). 
Table 2 Prevalence of diabetic retinopathy (DR) in patients diagnosed with diabetes mellitus after the age of 70. Association between DR and potential predictive variables

\begin{tabular}{|c|c|c|c|}
\hline & $\begin{array}{l}D R(+) \\
(n=21)\end{array}$ & $\begin{array}{l}D R(-) \\
(n=129)\end{array}$ & Significance level \\
\hline \multicolumn{4}{|c|}{ Duration of diabetes mellitus (years): } \\
\hline Median & 5.0 & 3.5 & \multirow[t]{2}{*}{$\mathrm{p}<0.007$} \\
\hline Range & $2.0-10.0$ & $0.5-16.4$ & \\
\hline \multicolumn{4}{|l|}{ Treatment regimen (n (\%)): } \\
\hline Insulin & $3(14)$ & $3(2)$ & \multirow{3}{*}{$\mathrm{p}<0.05$} \\
\hline Oral agents & $12(57)$ & $67(52)$ & \\
\hline Diet only & $6(33)$ & $59(45)$ & \\
\hline $\mathrm{HbA}_{1 \mathrm{c}}$ levels (\%) (mean (SD)) & $7.49(1.45)$ & $7.08(1.15)$ & NS \\
\hline \multicolumn{4}{|l|}{$\mathrm{HbA}_{1 \mathrm{c}}$ levels $(\mathrm{n}(\%))$ : } \\
\hline$>7 \%$ & $11(61)$ & $65(51)$ & \multirow[t]{2}{*}{ NS } \\
\hline$<7 \%$ & $7(39)$ & $62(49)$ & \\
\hline Hypertension (n (\%)) & $11(58)$ & $60(47)$ & NS \\
\hline \multicolumn{4}{|l|}{$\operatorname{Sex}(n(\%))$} \\
\hline Male & $8(38)$ & $50(39)$ & \multirow[t]{2}{*}{ NS } \\
\hline Female & $13(68)$ & $79(61)$ & \\
\hline
\end{tabular}

$\mathrm{DR}(+)=$ patients with $\mathrm{DR} ; \mathrm{DR}(-)=$ patients without $\mathrm{DR}$.

Table 3 Prevalence of diabetic retinopathy (DR) in patients with diabetes mellitus diagnosed after the age of 70. Comparison of data from this study and previously published studies

\begin{tabular}{|c|c|c|c|c|}
\hline & This study & $W E S D R^{3}$ & Nathan $^{5}$ & Segato $^{16}$ \\
\hline Age range studied (years) & $>70$ & $>70$ & $55-75$ & $>70$ \\
\hline No of patients in that age range & 150 & 151 & 185 & Data not available \\
\hline Age of patients at diagnosis & $>70$ & $>70$ & Variable & Variable \\
\hline \multicolumn{5}{|l|}{$\begin{array}{l}\text { Duration of diabetes mellitus } \\
\text { (years) }(\mathrm{n}(\%)) \text { : }\end{array}$} \\
\hline $0-4$ & $89(9)$ & $76(32)$ & & (18) \\
\hline $0-5$ & $110(12)$ & & $81(12)$ & \\
\hline $5-10$ & $55(24)$ & & & (31) \\
\hline $6-10$ & $34(24)$ & & $47(12)$ & \\
\hline $5-14$ & $59(20)$ & $66(35)$ & & \\
\hline $11-15$ & $4 \quad(0)$ & & $33(60)$ & \\
\hline $11-20$ & $6(0)$ & & & $(40)$ \\
\hline$>15$ & $2(0)$ & $9(55.6)$ & $24(48)$ & \\
\hline$>20$ & & & & (54) \\
\hline Overall prevalence of DR (\%) & 14 & 39 & 25 & 24.6 \\
\hline
\end{tabular}

ASSOCIATION BETWEEN DIABETIC RETINOPATHY AND $\mathrm{HbA}_{1 \mathrm{C}}$ LEVELS, SYSTEMIC HYPERTENSION, AND SEX OF PATIENTS

There was no significant difference between mean $\mathrm{HbA}_{1 \mathrm{c}}$ levels, or in the proportions of each group with $\mathrm{HbA}_{1 \mathrm{c}}$ levels above and below $7 \%$. Similarly, there was no difference in the proportions with and without hypertension, nor was there any association between DR and sex (Table 2).

COMPARISON OF PREVALENCE OF DIABETIC RETINOPATHY WITH PREVIOUSLY DETERMINED PREVALENCE DATA

For the purposes of comparing prevalence of DR according to differing durations of diabetes mellitus, duration of disease in this cohort has been categorised. Table 3 compares the prevalence of DR between this study and three previous studies representing different populations and regions. The Wisconsin Epidemiologic Study of Diabetic Retinopathy (WESDR) ${ }^{3}$ is a long term, population based study and includes a subgroup of patients diagnosed after the age of 70. The study of Nathan $e t a l^{5}$ is a hospital based study from the east coast of the USA, while that of Segato $e t a l^{16}$ is a population based study set in the northern region of Italy. Both of these studies include patients with varying ages at the time of diagnosis of diabetes. The current study, in common with these three studies, examines the association between prevalence of DR and duration of diabetes mellitus, mode of treatment, $\mathrm{HbA}_{1 \mathrm{c}}$ levels, presence of systemic hypertension, and sex of patients. The rates reported from the WESDR are considerably higher than rates reported from this study or the studies of Nathan et $a l^{5}$ and Segato et al. ${ }^{16}$ The differences in overall rates are also reflected in differences in duration of diabetes mellitus.

\section{Discussion}

While the present study concentrates on the prevalence of DR in patients diagnosed as having diabetes after the age of 70 years, three previous studies do have valuable data on the prevalence of DR in type II diabetics diagnosed with the disease in old age. ${ }^{3516}$ The main discrepancy between the four studies is in the presentation of the data on the association between prevalence of DR and duration of disease. This difference has already been highlighted in the results section.

The overall prevalence of DR in the patients in this study $(14 \%)$, was considerably lower than the overall prevalence seen in the WESDR $(40 \%)^{3}$ and lower than the overall prevalence in the data of Nathan et al $(26 \%)^{5}$ and Segato et al $(26.2 \%) .{ }^{16}$ Seven field stereographic fundal photographs (as used in the WESDR) and fluorescein angiography are the gold standard methods to detect DR. ${ }^{18}$ Experienced ophthalmologists using clinical examination methods (as employed in this study) may underestimate the prevalence of any DR, clinically significant macular oedema, and proliferative retinopathy by up to $20 \% .^{19-21}$ We do not feel, however, that examiner underestimation of prevalence by $20 \%$ can account for the disparity between the prevalence findings in the two studies $(14.0 \% \mathrm{v}$ $40.0 \%)$. The difference may be explained by the fact that our data come from a hospital based study, whereas the WESDR is a population based study. Alternatively, a much higher percentage of these elderly type II patients in the WESDR required insulin therapy $(50 \%$ compared with $4 \%$ ) which may indicate poorer diabetic control. Although Nathan $e t a \bar{P}$ and Segato et $a l^{16}$ used clinical examinations to detect DR, the selected groups of patients in these studies were not uniform with respect to age at the time the diagnosis of diabetes was made. The moderate difference between the overall prevalence figure reported in the present study and the figures reported in these studies may reflect the fact that within the spectrum of type II diabetes, the majority of elderly patients (>70 years) with no retinopathy at diagnosis do not have sufficient time to develop complications compared with their younger counterparts.

The present study has found, like earlier studies, that duration of diabetes was significantly associated with increased prevalence of DR. However, despite this finding, a number of patients had evidence of retinopathy at the time of diagnosis. It is accepted that some type II diabetics may have an indefinite period of asymptomatic hyperglycaemia before presentation ${ }^{3}$ and this may explain why a small percentage of patients in this study had visually threatening retinopathy at the time the diagnosis of diabetes was made. Two previous studies have noted that type II diabetics may have DR at 
presentation but the prevalence again was higher than that found by this study $(7 \%$ and $20 \%)^{22}{ }^{23}$ Asymptomatic hyperglycaemia before presentation may also account for the fact that when our duration data were categorised, all patients with sight threatening retinopathy had disease duration of less than 10 years. Segato et $a l^{16}$ have reported that the highest prevalence of proliferative retinopathy with disease duration of less than 10 years was seen in type II diabetics, although the patients in that study were heterogeneous with respect to age at diagnosis. The present study, unlike the three studies used for comparison, ${ }^{3516} \mathrm{did}$ not find a linear relation between duration of diabetes and prevalence of DR after 10 years of diabetes. Owing to its small size $(n=11)$, the group with this disease duration in our study may be an unrepresentative sample but the natural mortality rate of people older than 80 $(>70$ years plus 10 years) may explain the low numbers of elderly type II diabetics with diabetes for more than 10 years. Likewise, the presence of DR is often associated with systemic complications and resulting higher mortality rates, which could account for the very low prevalence of DR associated with duration of 10 years or more seen in this group.

Our study demonstrates that patients on insulin had a three and a half times higher prevalence of retinopathy compared with patients on oral hypoglycaemic agents and a five times higher prevalence compared with those patients on diet alone, a finding supported by previous studies. ${ }^{2} 3516$ Requiring insulin for control in type II diabetes may reflect poor diabetic control and thus persistent hyperglycaemia, while reduction of hyperglycaemia reduces the risk of developing DR and slows its progression. ${ }^{24}$ It is also possible that insulin use itself may have a direct association with retinopathy. ${ }^{16}$

The current study, in contrast with other studies, ${ }^{3516}$ did not show an association between an increased prevalence of DR and elevated serum $\mathrm{HbA}_{1 \mathrm{c}}$ levels in patients with type II disease. The mean $\mathrm{HbA}_{1 \mathrm{c}}$ level of those patients with some form of retinopathy was insignificantly higher than the mean level of the group without retinopathy, but both groups' means were above the clinics' normal levels. This study used a more homogeneous patient group than other studies which have included patients with a range of ages. ${ }^{3516}$ The overall lower prevalence of retinopathy in these older patients even with a mean $\mathrm{HbA}_{1 \mathrm{c}}$ level above normal suggests that a $\mathrm{HbA}_{1 \mathrm{c}}$ of less than $7.0 \%$ may be too strict a target for these older patients as a general rule.

The study reported in this paper found no association between systemic hypertension and an increased prevalence of DR. While earlier reports were inconclusive regarding the association between systemic hypertension and $\mathrm{DR},{ }^{3}$ later specific studies found no relation between hypertension and retinopathy in type II diabetics. ${ }^{51625}$

The clinical implications for these elderly diabetic patients diagnosed after the age of 70 years is that they should have a thorough eye examination near to the time the diagnosis of diabetes is made, to determine whether or not any DR is present. Our prevalence data demonstrate that the majority will have no retinopathy and that the majority with retinopathy, have non-sight threatening background retinopathy. However, it is important that the small percentage of patients with retinopathy at the time of diagnosis of diabetes are detected and, if necessary, treated. Patients with longer duration of diabetes and who require insulin (which may indicate poor dietary compliance and poor diabetic control), have the highest prevalence of retinopathy. While it may appear that the majority of patients will not have any retinopathy, all of these patients require regular follow up. The practice in our regional centre is that follow up in patients without retinopathy is every 18 months to 2 years, but an incidence study of retinopathy is required in this group of elderly patients to determine an accurate figure.

\section{Conclusions}

The overall prevalence of DR in type II diabetics diagnosed after the age of 70 is lower than that previously reported in cohorts of patients with type II disease with a range of ages at diagnosis. While the majority of elderly type II diabetics (greater than 70 years at diagnosis) will not develop significant DR, a small percentage of patients will have visually threatening retinopathy at presentation. Furthermore, type II diabetic patients diagnosed after the age of 70 with longer disease duration and requiring insulin have a significantly increased prevalence of DR. The clinical implication is that all elderly diabetics should have a thorough ophthalmic examination near to the time of first presentation and regular ophthalmic examinations thereafter.

1 Retinopathy Working Party. A protocol for screening for diabetic retinopathy in Europe. Diabetic Med 1991;8:263-7. 2 Klein R, Klein BEK, Moss SE, Davis MD, DeMets DL. The Kein R, Klein BEK, Moss SE, Davis MD, DeMets DL. The Prevalence and risk of diabetic retinopathy when age at Prevalence and risk of diabetic retinopathy when age at
diagnosis is less than 30 years. Arch Ophthalmol 1984;102: $520-6$

3 Klein R, Klein BEK, Moss SE, Davis MD, DeMets DL. The Wisconsin Epidemiologic Study of Diabetic Retinopathy. III Prevalence and risk of diabetic retinopathy when age at diagnosis is 30 or more years. Arch Ophthalmol 1984;102: 527-32.

4 Yanko L, Goldbourt U, Michaelson IC, Shapiro A, Yaari S. Prevalence and 15 year incidence of retinopathy and associated characteristics in middle aged and elderly diabetic men. Br f Ophthalmol 1983;67:759-65.

5 Nathan DM, Singer DE, Godine JE, Hodgson Harrington C, Permulter LC. Retinopathy in older type II diabetics: association with glucose control. Diabetes 1986;35:797801 .

6 Agardh E, Torffvit O, Agardh CD. The prevalence of retinopathy and associated medical risk factors in type I (insulin-dependent) diabetes mellitus. F Intern Med 1989; (insulin-depen

7 Gall MA, Rossing P, Skott P, Damsbo P, Vaag A, Bech K, et al. Prevalence of micro- and macroalbuminuria, arterial hypertension, retinopathy and large vessel disease in European type II (non-insulin-dependent) diabetic patients. Diabetologia 1991;34:655-61.

8 Jerneld B, Algerve P. Relationship of duration and onset of diabetes to prevalence of diabetic retinopathy. $A m \mathcal{F}$ Ophthalmol 1986;102:431-7.

9 Ishihara M, Yukimura Y, Yamada T, Ohto K, Yoshizawa K. Diabetic complications and their relationships to risk factors in a Japanese population. Diabetes Care 1984;7:533-8.

10 Diabetes Drafting Group. Prevalence of small vessel and large vessel disease in diabetic patients from 14 centers: the arge vessel disease in diabetic patients from 14 centers: the World Health Organisation Multinational Study of Va

11 Hafner SM, Fong D, Stern MP, Pugh JA, Hazuda HP Patterson JK, et al. Diabetic retinopathy in Mexican Americans and non Hispanic whites. Diabetes 1988;37:878-84. 
12 Nelson RG, Wolfe JA, Horton MB, Pettitt DJ, Bennett PH, Knowler WC. Proliferative retinopathy in NIDDM. 38:435-40.

13 Hamman RF, Mayer EJ, Moo-Young GA, Hilderbrandt W, Marshal JA, Baxter J. Prevalence and risk factors of diabetic retinopathy in non Hispanic whites and Hispanics with NIDDM. San Luis Valley Diabetes Study. Diabetes 1989;38:1231-7.

14 Kahn HA, Bradley RF. Prevalence of diabetic retinopathy. Age, sex and duration of diabetes. $\mathrm{Br} \mathcal{f}$ Ophthalmol 1975;59:345-9.

15 Mouton DP, Gill AJ. Prevalence of diabetic retinopathy and evaluation of risk factors. A review of 1005 diabetic clinic patients. S Afr Med f 1988;74:399-402.

16 Segato I, Midena E, Grigoletto F, Zucchetto M, Fedele D, Piermarocchi S, et al. The epidemiology and prevalence of diabetic retinopathy in the Veneto region of north east Italy. Diabetic Med 1991;8 Sym:S11-6.

17 Health Statistics 1993. Dublin: Department of Health, Government Publications Office.

18 Diabetes Control and Complications Trial Research Group. Color photography vs fluorescein angiography in the detection of diabetic retinopathy in the diabetes control and complications trial. Arch Ophthalmol 1987;105:1344-51.

19 Schachat AP, Hyman L, Leske MC, Connell AM, Hiner C, Javornik $\mathrm{N}$, et al. Comparison of diabetic retinopathy detection by clinical examinations and photograph gradings. Arch Ophthalmol 1993;111:1064-70.

20 Kinyoun J, Barton F, Fisher M, Hubbard L, Aiello L, Ferris F. Detection of diabetic macular oedema. Ophthalmoscopy versus photography-Early Treatment Diabetic Retinopathy Study Report Number 5. The ETDRS Study Group. Ophthalmology 1989;96:746-51.

21 Moss SE, Klein R, Kessler SD, Richie KA. Comparison between ophthalmoscopy and fundus photography in determining severity of diabetic retinopathy. Ophthalmology 1985;92:62-7.

22 Soler NG, Fitzgerald MG, Malins GM, Summers R. Occurrence of retinopathy in early diabetes mellitus. BMF 1969; 3:567-9.

23 Klein R, Klein BEK, Moss SE. Prevalence of diabetes mellitus in southern Wisconsin. Am f Epidemiol 1984;119:5461

24 Diabetes Control and Complications Trial Research Group. The effect of intensive treatment of diabetes on the development and progression of long term complications in insulin-dependent diabetes mellitus. $N$ Engl $f \mathrm{Med}$ 1993;329:977-86.

25 Klein R, Klein BE, Moss SE, Davis MD, DeMets DL. Is blood pressure a predictor of the incidence or progression of diabetic retinopathy? Arch Intern Med 1989;149:242732 